\title{
Reduction of Nonlinear Intrachannel Effects by Channel Asymmetry in Transmission Lines With Strong Bit Overlapping
}

\author{
E. G. Shapiro, M. P. Fedoruk, S. K. Turitsyn, and A. Shafarenko
}

\begin{abstract}
We have examined the statistics of simulated bit-error rates in optical transmission systems with strong patterning effects and have found strong correlation between the probability of marks in a pseudorandom pattern and the error-free transmission distance. We discuss how a reduced density of marks can be achieved by preencoding optical data.
\end{abstract}

Index Terms-Forward-error correction (FEC), numerical analysis, optical fiber communication, run length codes.

\section{INTRODUCTION}

O PTIMIZATION of the optical transmission system parameters is a crucial task for the design of fiber links. Numerical evaluation of system performance in terms of error probability is still in progress, especially in the presence of strong patterning effects (SPE), when the simulated transmission distance greatly varies from run to run. For high bit-rate dispersion-managed fiber systems with many bits significantly overlapping during transmission, the patterning effects are quite significant. Consequently, the conventional approach [1] needs to be modified to account for statistical variations of the computed $Q$ factor for different patterns used in simulations [2], [3]. In this letter, we study the statistics of the numerically calculated $Q$ factor under SPE and examine the suppression of intrachannel nonlinear effects when the probability of a mark decreases.

\section{A. Statistics of the $Q$ Factor}

For the sake of simplicity, but without loss of generality, we assume, following [1], that the statistics of the received marks and spaces can be approximated by two Gaussian distributions: $p_{1}(x)=\exp \left[-\left(x-\overline{\mu_{1}}\right)^{2} / 2{\overline{\sigma_{1}}}^{2}\right] /\left(\overline{\sigma_{1}} \sqrt{2 \pi}\right)$ and $p_{0}(y)=\exp \left[-\left(y-{\overline{\mu_{0}}}^{2} / 2{\overline{\sigma_{0}}}^{2}\right] /\left(\overline{\sigma_{0}} \sqrt{2 \pi}\right)\right.$, respectively. The $Q$ factor is then defined as $Q=\left(\overline{\mu_{1}}-\overline{\mu_{0}}\right) /\left(\overline{\sigma_{1}}+\overline{\sigma_{0}}\right)$. Consider varying patterns of $N=n_{1}+n_{0}$ bits where $n_{1}$ and $n_{0}$ are the

Manuscript received December 6, 2002; revised June 13, 2003. This work was supported by the Russian Foundation for Basic Research under Grant 03-02-16 496-a.

E. G. Shapiro is with the Institute of Aautomation and Electrometry, Russian Academy of Science, 630090 Novosibirsk, Russia.

M. P. Fedoruk is with the Institute of Computational Technologies, Russian Academy of Science, 630090 Novosibirsk, Russia.

S. K. Turitsyn is with the Photonics Research Group, School of Engineering and Applied Science, Aston University, Birmingham B4 7ET, U.K. (e-mail: s.k.turitsyn@aston.ac.uk).

A. Shafarenko is with the Department of Computer Science, University of Hertfordshire, Hatfield AL10 9AB, U.K. (e-mail: a.shafarenko@ herts.ac.uk).

Digital Object Identifier 10.1109/LPT.2003.818061 numbers of marks and spaces. Under SPE, $Q$ varies from pattern to pattern and, in general, differs from the value of the "true" $Q$ for a very large (or infinite) pattern. In practice, it is important to understand how the $Q$ factor found from a finite-length pattern correlates with the one corresponding to the infinite pattern. Let $x_{1}, x_{2}, \ldots, x_{n 1}$ and $y_{1}, y_{2}, \ldots y_{n 0}$ be the simulated values of marks and spaces, respectively. Define, following [4], the sample $\mu_{1}, \mu_{0}$ and $\sigma_{1}, \sigma_{0}$ for these and consider the distribution of a random quantity $Q_{n_{1}+n_{0}}=\left(\mu_{1}-\mu_{0}\right) /\left(\sigma_{1}+\sigma_{0}\right)$. Assuming that $\mu_{0}$ and $\mu_{1}$ are statistically independent, $\mu_{1}-\mu_{0}$ is also normal distributed, with the standard deviation $\sigma=\sqrt{{\overline{\sigma_{1}}}^{2} / n_{1}+{\overline{\sigma_{0}}}^{2} / n_{0}}$ and the mean $\mu=\overline{\mu_{1}}-\overline{\mu_{0}}$. Further, $1 /\left({\overline{\sigma_{1}}}^{2}\right) \sum_{k=0}^{n_{1}}\left(x_{k}-\mu_{1}\right)^{2}=\left(\left(n_{1}-1\right) \sigma_{1}^{2}\right) /\left({\overline{\sigma_{1}}}^{2}\right)$ is distributed by $\chi_{n_{1}-1}^{2}$ (chi-squared distribution with $n_{1}-1$ degrees of freedom). Similarly, $\left(n_{0}-1\right) \sigma_{0}^{2} /{\overline{\sigma_{0}}}^{2}$ is distributed by $\chi_{n_{0}-1}^{2}$. We aim to estimate the probability that random $Q_{n_{1}+n_{0}}=\left(\mu_{1}-\mu_{0}\right) /\left(\sigma_{1}+\sigma_{0}\right)$ will be larger/smaller than the "true" $Q$ factor $Q=\left(\overline{\mu_{1}}-\overline{\mu_{0}}\right) /\left(\overline{\sigma_{1}}+\overline{\sigma_{0}}\right): \operatorname{Pr}\left[\left(\mu_{1}-\mu_{0}\right) /\left(\sigma_{1}+\right.\right.$ $\left.\left.\sigma_{0}\right)>Q\right]=\operatorname{Pr}\left[\mu_{1}-\mu_{0}-\left(\overline{\mu_{1}}-\overline{\mu_{0}}\right) /\left(\overline{\sigma_{1}}+\overline{\sigma_{0}}\right)\left(\sigma_{1}+\sigma_{0}\right)>0\right]$. Note that for systems with $\sigma_{1} \gg \sigma_{0}$, we can approximate the $Q$ factor by $Q_{n_{1}+n_{0}}^{\prime}=\left(\mu_{1}-\mu_{0}\right) / \sigma_{1}$. The variable $Q_{n_{1}+n_{0}}^{\prime}$ is distributed by $1 /\left(\sqrt{n_{1}}\right)(\mu / \sigma+U) /\left(\chi_{n_{1}-1} / \sqrt{n_{1}-1}\right)$ (where $U$ is the standard normal distribution and $\left.\mu=\mu_{1}-\mu_{0}\right)$, which is the noncentral $T$-student distribution with $n_{1}-1$ degrees of freedom. Then for large $n_{1}$, we can use the known fact [4] that the distribution of $\sinh ^{-1}\left[Q^{\prime} \sqrt{3 / 2}\right]$ approximates normal with the mean value $(\mu / \sigma) \sqrt{3 /\left(2 n_{1}\right)}$ and dispersion $\sqrt{3 /\left(2 n_{1}\right)}$. When both $n_{1}$ and $n_{0}$ are large, we can greatly simplify analysis by applying the known result that each of $\sqrt{2 \chi_{n_{1}-1}^{2}}-\sqrt{2\left(n_{1}-1\right)-1}$ and $\sqrt{2 \chi_{n_{0}-1}^{2}}-\sqrt{2\left(n_{0}-1\right)-1}$ is well approximated by the standard normal distributions (the so-called Fisher's approximation [4]). Therefore, in the limit of large $n_{1}$ and $n_{0}, \mu_{1}-\mu_{0}-\left(\overline{\mu_{1}}-\overline{\mu_{0}}\right) /\left(\overline{\sigma_{1}}+\overline{\sigma_{0}}\right)\left(\sigma_{1}+\sigma_{0}\right)$ approximates a normal distribution with the mean value $\left(\overline{\mu_{1}}-\overline{\mu_{0}}\right) /\left(\overline{\sigma_{1}}+\overline{\sigma_{0}}\right)\left[\left(\overline{\sigma_{1}}\right) /\left(4\left(n_{1}-1\right)\right)+\left(\overline{\sigma_{0}}\right) /\left(4\left(n_{0}-1\right)\right)\right]$ which obviously tends to zero as $n_{1}, n_{0} \rightarrow \infty$. This result implies that the probability for the $Q$ factor $Q_{n_{1}+n_{0}}$ of a random pattern to be larger than the "true" $Q$ tends to 0.5 , i.e., that overestimates and underestimates of $Q$ are equally probable. System performance is usually analyzed in terms of maximum propagation distance corresponding to a BER less than $10^{-9}$ (or $Q>6$ ). Consider $M$ sample calculations of $Q$ factor for which error-free (with $Q>6$ ) transmission distances have been calculated and arranged in ascending order: $Z_{1}<Z_{2}<\cdots<Z_{M}$. It follows from the above result that the 
TABLE I

PARAMETERS OF THE SYSTEM

\begin{tabular}{l|c|c}
\hline Fibre parameters & SMF & DCF \\
\hline \hline Length $\mathrm{km}$ (amplifier spacing) & 32.485 & 6.356 \\
\hline Dispersion D at $1553 \mathrm{~nm} \mathrm{ps} /(\mathrm{km} \cdot \mathrm{nm})$ & +16.4 & -84 \\
\hline Dispersion slope $\mathrm{dD} / \mathrm{d} \lambda \mathrm{ps} /\left(\mathrm{km} \cdot \mathrm{nm}^{2}\right)$ & +0.06 & -0.3 \\
\hline Loss $\mathrm{dB} / \mathrm{km}$ & 0.21 & 0.5 \\
\hline Nonlinear refractive index $n_{2} 10^{-20} \cdot \mathrm{m}^{2} / W$ & 2.67 & 2.67 \\
\hline Effective fiber area $A_{\text {eff }} \mu m^{2}$ & 80 & 26 \\
\hline
\end{tabular}

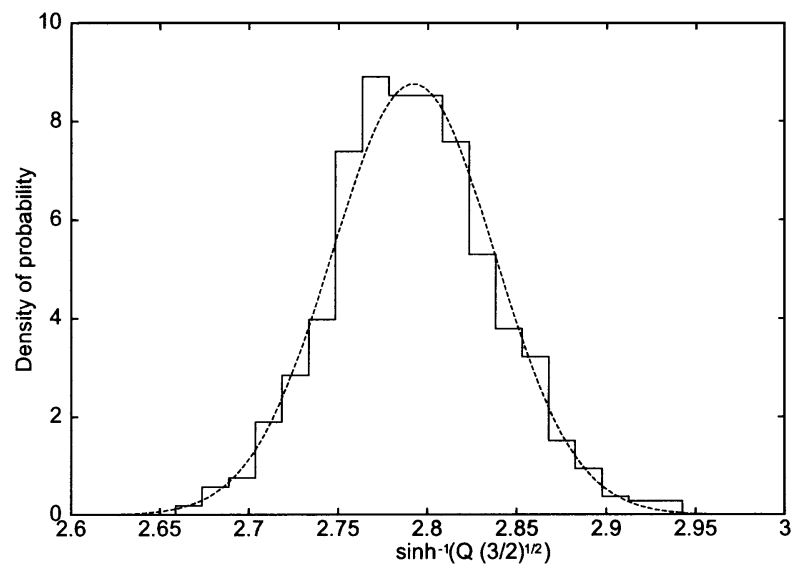

Fig. 1. Distribution of $\sinh ^{-1}[Q \sqrt{3 / 2}]$.

sample with the median value in this sequence gives the best approximation of the "true" error-free distance.

\section{B. Numerical Modeling}

For the purposes of verification of the above theory, we chose a symmetric dispersion map. The periodic cell starts with the standard fiber (SMF) followed by the dispersion compensating fiber (DCF), then again by the piece of SMF (of the same length as the first SMF), and finally by $10-\mathrm{dB}$ EDFA amplifier. The fiber parameters are listed in Table I.

Average dispersion was $-0.03 \mathrm{ps} / \mathrm{nm} / \mathrm{km}$. Parameters of the input unchirped Gaussian pulses are: width: $6.7 \mathrm{ps}$; peak power: $4.95 \mathrm{~mW}$. We have examined the statistics of four-channel wavelength-division-multiplexing (WDM) transmission with $150-\mathrm{GHz}$ channel separation at $40 \mathrm{~Gb} / \mathrm{s}$ in each channel. The highest frequency channel was operating at $1548.2 \mathrm{~nm}$. Instant detection at every numerical step was performed to locate the maximum performance points inside the map period. Fig. 1 presents the distribution of $\sinh ^{-1}[Q \sqrt{3 / 2}]$ calculated after 350 runs with random patterns of $512 \mathrm{~b}$; here, $Q$ is taken at $Z=2875 \mathrm{~km}$ in the worst of the four WDM channels. In agreement with the above theory, the distribution approximates normal (the probability of a type 1 error for this from the $\chi^{2}$ test is 0.02 ). There is a patterning effect in the system due to large bit overlapping. This leads to a strong correlation between the number of marks in the pattern and degradation of the signal. Fig. 2 shows the correlation coefficient (see, e.g., [4]) between the number of marks in the pseudorandom pattern and the value of $Q$. Negative correlation coefficient indicates that the degradation of the $Q$ factor is related to the number of marks in the pattern.

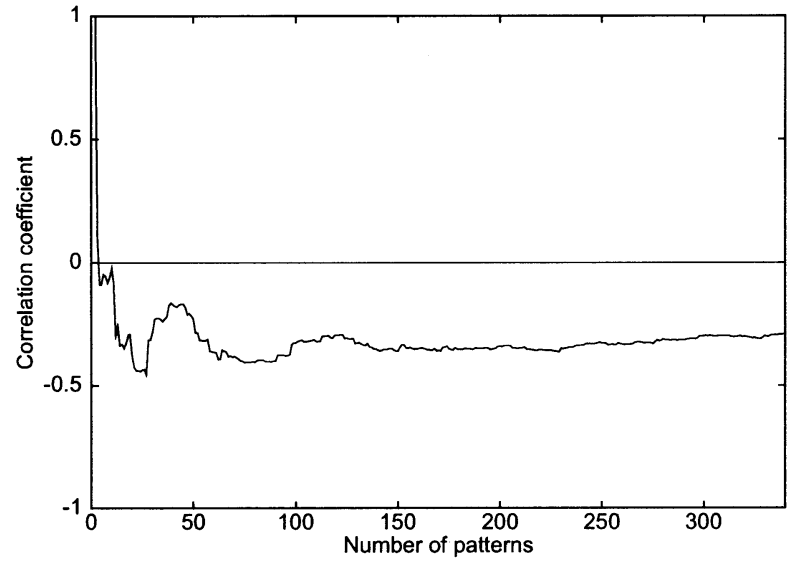

Fig. 2. Correlation between the number of marks in the pattern and the value of $Q$ factor.

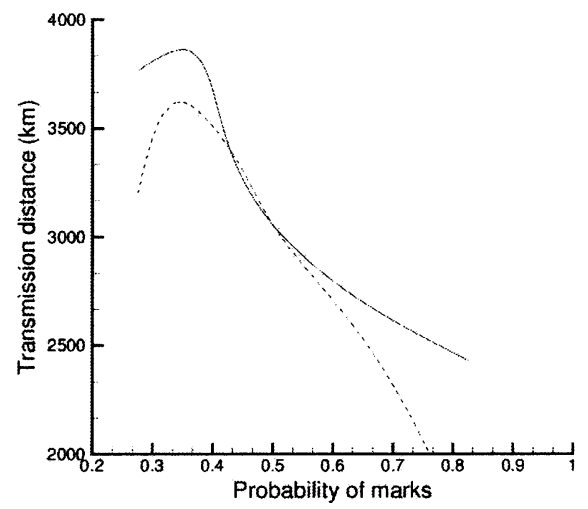

Fig. 3. Effect of the probability of marks on transmission distance (the dashed curve is bandwidth-adjusted).

Fig. 3 shows how the error transmission distance decreases with the rising probability of marks in the pattern. The information content of a binary digit is $W(p)=-p \log _{2} p-(1-$ $p) \log _{2}(1-p)$, where $p$ is the probability of marks. In particular, when marks and spaces are equally probable the information content of one binary digit is exactly equal to $1 \mathrm{~b}$. As the value of $p$ deviates from $1 / 2$, the information content drops roughly as the square of the deviation. The information content of a string of bits is proportional to its length and to the content of each bit, assuming the absence of correlations. Hence, the product $M(p)=W(p) \mathrm{TD}(p)$ (here, TD is error-free transmission distance) is proportional to the standard figure of merit for fiber lines (bandwidth times distance). Furthermore, $M(p)$ has the natural meaning of "bandwidth-adjusted" transmission distance.

Note that as the probability of marks decreases, so too does the average energy contained in the packet. To compensate for this (and ensure a constant level of nonlinearity in the system for all patterns), the energy of each mark was proportionally increased. This explains why the unadjusted curve bends down at low probabilities of marks. This effect accentuates the maximum in the bandwidth-adjusted curve, where a similar degradation occurs due to the information content going down as the contrast between mark and space probabilities increases. There is, thus, a well-defined optimal encoding regime for the transmission system in question. 
Discussion: The results of our simulations indicate that the density of marks in the message dramatically affects the quality of transmission. It is, therefore, desirable to preencode the message in such a way that the number of marks is kept near optimum before transmission. This can be achieved by bit stuffing, i.e., by inserting additional spaces, which are to be discarded, or "unstuffed," by the receiver. The insertion of the spaces could be in fixed randomly chosen positions, in which case the sequence remains uncorellated as a whole, or, rather more promisingly, in those positions where the local density of marks exceeds a certain limit. In the latter case, we introduce certain correlations, which are not covered by the transmission analysis presented above directly; however, it stands to reason that a system with local interactions between pulses should respond to local bursts of load as well. In the case of fixed insertion positions, extra spaces will often be inserted into a run of spaces where they do not affect the physics of transmission and so could well be wasted.

Targeted insertion of spaces is well known in digital recording where it is referred to as run-length limited (RLL) encoding. The RLL is applied as a modulation technique which prevents runs of marks exceeding a certain, usually small, length from occurring on the recorded medium. It supports very efficient encoding-decoding solutions based on linear complexity algorithms. The downside of the additional correlation that results from RLL is not only the need to further investigate the propagation of correlated (as opposed to uncorrelated) sequences, it is also the fact that RLL decoding can proliferate errors by misinterpreting faulty RLL-encoded fragments. Thus, single transmission errors, which could easily be corrected by uncomplicated forward-error correction (FEC) methods, may well be turned into harder-to-correct burst errors by the RLL decoder.

There is consequently a need for a combined RLL-FEC preencoding, which provides sufficient space stuffing while keeping possible transmission errors under control. In a recent paper [8], such codes have been proposed. They are free from induced burst errors, amenable to an efficient hardware implementation, and can accommodate any external FEC code (which consequently does not have to be burst-correcting). In particular, the FEC code could be one of the low-density parity check codes (see [5]-[7]), which are known for their high performance [5] as well as the fact that they lend themselves nicely to a parallel implementation.

Future work will focus on studying the propagation of correlated sequences under RLL-FEC with the view to quantifying the effects described here and providing further recommendations on the optimal encoding regime.

\section{REFERENCES}

[1] N. S. Bergano, F. W. Kerfoot, and C. R. Davidson, "Margin measurements in optical amplifier system," IEEE Photon. Technol. Lett., vol. 5, pp. 304-306, Mar. 1995.

[2] C. J. Anderson and J. A. Lyle, "Technique for evaluating system performance using $Q$ in numerical simulations exhibiting intersymbol interference," Electron. Lett., vol. 30, pp. 71-72, 1994.

[3] E. G. Shapiro, M. P. Fedoruk, and S. K. Turitsyn, "Numerical estimate of BER in optical systems with strong patterning effects," Electron. Lett., vol. 37, pp. 1179-1181, 2001.

[4] N. J. Johnson and S. Kotz, Distributions of Statistics. New York: Wiley, 1972.

[5] D. J. C. MacKay and R. M. Neal, "Near Shannon limit performance of low density parity check codes," Electron. Lett., vol. 32, no. 18, pp. 1645-1646, 1996.

[6] M. Sipser and D. Spielman, "Expander codes," IEEE Trans. Inform. Theory, vol. 42, no. 6, pp. 1710-1722, Nov. 1996.

[7] M. C. Davey and D. J. C. MacKay, "Low-density parity check codes over GF(q)," IEEE Commun. Lett., vol. 2, pp. 165-167, June 1998.

[8] A. J. van Wijngaarden and K. A. S. Immink, "Maximum runlength-limited codes with error control capabilities," IEEE J. Select. Areas Commun., vol. 19, pp. 602-611, Apr. 2001. 